\title{
Confirmatory factor analyses of the Parent Attributions Questionnaire among Asian immigrant mothers
}

\author{
By Huiguang Ren, Shuyan Sun, Charissa S. L. Cheah, Biao Sang, and Junsheng Liu
}

\begin{abstract}
The present study examined the factor structure of maternal attributions regarding their caregiving experiences, measured by the Parent Attributions Questionnaire, among Asian immigrant mothers. Chinese and Korean Immigrant mothers $\left(N=333, M_{\text {age }}=36.79\right.$ years, $S D=$ 4.79) with preschool children $\left(M_{a g e}=4.43\right.$ years, $\left.S D=1.31\right)$ reported their attributions regarding the reasons for their success and failure at seven daily caregiving tasks, and demographic information. Three structure models were tested: the unidimensional structure model, the internal-external structure model, and the controllable-uncontrollable structure model. Results revealed that the controllability-based structure fitted the data better than the unidimensional or locus-based structure of parental attributions. Metric and partial scalar invariance of the controllability-based model were established between Chinese and Korean immigrant mothers. Asian immigrant mothers focused on whether the causes for the parenting outcomes were controllable or uncontrollable when attributing the causes of their caregiving experiences. The superiority of the controllable-uncontrollable over the internal-external structure model in this sample may reflect Asian immigrant mothers’ Confucian-based heritage cultural emphasis on their responsibility for creating optimal childrearing for their children.
\end{abstract}


Keywords: Asian immigrant mothers, parental attribution, confirmatory factor analysis, measurement invariance, controllability attributions, Parent Attributions Questionnaire 
Confirmatory Factor Analyses of the Parent Attributions Questionnaire among Asian Immigrant

\section{Mothers}

Parental attributions are parents’ causal interpretations of their own' behaviors, their children’s behaviors and parent-child interactions (Sigel, McGillicuddy-DeLisi, \& Goodnow, 2014). Parents' attributions influence their emotional and behavioral reactions to their children, and can further lead to children's social (e.g., social withdrawal, aggression) and cognitive (e.g., academic achievement) outcomes (Enlund, Aunola, \& Nurmi, 2015; Miller, 1995). Despite the large and growing Asian immigrant population in the U.S., (Humes, Jones, \& Ramirez, 2011), the parental attribution of Asian immigrant mothers and the measurement of this important aspect of parenting cognitions have received little attention, and thus, were the focus of the present study. The understanding of parenting attributions has been discussed as being important for modifying behavior in parent training programs (Sawrikar \& Dadds, 2018).

\section{The Parent Attributions Questionnaire}

The Parent Attributions Questionnaire (PAQ) is a widely used measure specifically developed to assess parents’ causal attributions for daily parenting tasks (MacPhee, Seybold, \& Fritz, n.d.; Sirignano \& Lachman, 1985). In the PAQ, parents are asked to rate how much their success and failure in seven daily parenting tasks (e.g., dressing, bathing) are due to each of 5 potential causes (parent’s ability, mood, effort, task difficulty, and children’s cooperation).

The parenting tasks in the measure are considered universal and familiar to mothers in most parts of the world (Bornstein et al., 1998). Thus, the PAQ has been widely used to measure maternal attributions and to examine associated outcomes of maternal attributions in many different samples (e.g., Bornstein \& Cote, 2004; Bornstein et al., 1998; Lee \& Han, 2017). 
However, despite its wide utilization, the factor structure of the PAQ has not yet been well-identified and demands further clarification, especially for non-Western groups.

\section{Locus-Based Factor Structure of the PAQ}

The PAQ was developed based on Weiner and colleagues' attribution theory (1971), which proposed two dimensions of causal attributions: locus and stability. Studies of parental attribution, particularly parents' explanation for their children's behaviors, usually utilize the locus dimension as a criterion to distinguish different types of causal interpretations (e.g., Cheah \& Rubin, 2003, 2004). Specifically, internal attributions for children’s behaviors refer to perceptions that children's behaviors are caused by dispositional (e.g., temperamental) and intentional sources. In contrast, external attributions refer to perceptions that children's behaviors are caused by situational and environmental forces (Dix \& Grusec, 1985).

Although the PAQ is a measure of parental attribution concerning parent-child interactions rather than children's behaviors, users of the PAQ still tend to embrace the locus-based theoretical structure (e.g., Bornstein \& Putnick, 2007; Bornstein et al., 2017). Of the five causes in this measure, three causes (i.e., parental effort, mood and ability) are considered internal parental attributions since they are situated within the parent. In contrast, the other two causes (i.e., task difficulty and child cooperation) are considered external parental attributions because these reasons are situated outside the parent, specifically in the child and task.

The locus-based structure of the PAQ is supported by a cross-national study, which demonstrated negative correlations between different causes as well as a unidimensional structure of the PAQ among 7 different countries (Bornstein et al., 1998). The unidimensional structure is further referred to as evidence for distinguishing between different attributions based on its locus (e.g., Bornstein \& Putnick, 2007; Bornstein et al., 2017). However, in Bornstein et 
al., (1998)'s cross-national study, the five causes were ranked from the most important to the least important rather than rated individually as a potential explanation, which likely accounted for the negative correlations between the different causes and the unidimensional structure. However, the PAQ was originally designed for parents to rate, rather than rank, the causes (Sirignano \& Lachman, 1985), and positive correlations rather than negative correlations between different causes were reported by the original studies (MacPhee et al., n.d.). With an increasing number of studies now utilizing ratings for the PAQ rather than rankings (e.g., Bornstein \& Putnick, 2007; Lee \& Han, 2017), the factor structure of ratings of the PAQ items deserves attention.

\section{Controllability-Based Factor Structure of the PAQ}

Weiner further modified his attribution theory to include a third dimension of controllability (Weiner, 1985). Specifically, controllable causes for parenting outcomes are perceived to be within parents' control (e.g., parents' efforts or mood), and thus, more malleable. In addition to parents' effort and mood, Asian parents may also view child cooperation as a controllable cause due to their general cultural focus on parental control and emphasis on the interdependence of family members, especially the subordinate roles of children to parents (Chao, 1995; Cheung \& Pomerantz, 2011; Park, Kim, Chiang, \& Ju, 2010). In contrast, uncontrollable attributions refer to causes that are out of parents’ control (e.g., parents’ ability or task difficulty), and stable and constant. Either the locus or controllability of a cause could be the primary focus depending on the content regarding which the parental attributions are made, and in some situations, whether a cause is controllable or not is proposed to be even more important than its locus (Yirmiya \& Weiner, 1986). The locus-based structure is more often examined in studies about parental attributions of children’s behaviors (e.g., Cheah \& Rubin, 2003, 2004; Snarr, Slep, 
\& Grande, 2009), whereas the controllability dimension is increasingly emphasized especially for parental attributions of their own parenting practices (e.g., Woolfson, Taylor, \& Mooney, 2011), likely because of the implications for changing parenting behavior. Nevertheless, the controllability-based structure has not been examined in the PAQ despite strong theoretical and empirical evidence for the relevance of a controllability dimension in parental attributions.

\section{East Asian Childrearing Values}

Chinese and Korean immigrants represent two of the largest East Asian immigrant populations in the United States (Humes et al., 2011). Mothers from China and Korea share some similar cultural backgrounds and childrearing attitudes due to their Confucian historical roots (Chao, 2000). This similarity has been found to persist even after the migration of these groups to the U.S., with research revealing some consistent patterns of parenting beliefs among immigrant mothers from China and Korea (Sung, 2010). For example, Chinese and Korean immigrant families in the U.S. emphasize collectivism, hierarchical parent-child relationships, and parental control over child autonomy, more strongly than their Western counterparts (Chao, 2001).

Collectivism and parental attribution. Collectivistic cultures within East Asian countries, including China and Korea, generally endorse in-group harmony and cohesion over individuality based on Confucian values (Triandis, 2001). This collectivism is also reflected in East Asian mothers’ childrearing values. For example, East Asian mothers emphasize interdependence among family members (Chao, 1995; Park et al., 2010) and view their children as more exposed and connected to environmental and external influences (Ho, 1986). Consistent with this idea, mothers from China and Korea consistently make more external causal attributions for their children's behaviors (e.g., social withdrawal, aggression) because of their 
cultural emphasis on environmental influences (Cheah \& Rubin, 2003, 2004; Park \& Cheah, 2005). However, when it comes to parent-child interactions, parents themselves become the major source of environmental influence on their children. Therefore, East Asian parents may focus primarily on whether they can control the causes of the childrearing outcomes instead of the locus of the causes. As a measure designed for parental attributions of parent-child interactions rather than children's behaviors, the PAQ may be more appropriately explained with the controllability-based instead of the locus-based structure for East Asian parents.

Parental control and parental attribution. Another important feature of East Asian cultures is the focus on control. Asian parents are more likely to emphasize parental control as a way of expressing love and fulfilling parental responsibilities whereas Western parents emphasize child autonomy (Chao, 2001; Pomerantz \& Wang 2009). Consistent with these different cultural emphases, some studies have found that Asian parents reported more parental control and engaged in more authoritarian and less authoritative parenting compared with Western parents (e.g., Cheung \& Pomerantz, 2011; Ng, Pomerantz, \& Deng, 2014). The cultural endorsement of parental control among Asian parents has been indicated in behavioral aspects of their parenting, but could also be reflected in their parenting cognitions. That is, Asian immigrant parents may evaluate and attribute parent-child interactions based on a controllability dimension because they may be culturally attuned to whether the causes of these events are within their control.

\section{The Present Study}

Previous research on parental attributions has focused more on parents’ attributions for their children’s behaviors, or child-referent attributions. Instead, parents’ attributions for their 
own parenting experiences, known as parent-referent attributions, have been less extensively examined in Asian families, particularly those that are residing in a Western context.

Therefore, we examined the factor structure of the PAQ in a sample of Chinese immigrant and Korean immigrant mothers to explore whether a unidimensional, locus-based, or controllability-based structure (see Figure 1) is more appropriate for the measure within these samples. There were three specific aims in the present study: (1) to confirm the factor structure of the PAQ in the Asian immigrant sample, (2) to establish the metric and scalar invariance between the Chinese immigrant and the Korean immigrant samples, and (3) to examine the reliability of the PAQ in the Chinese immigrant and Korean immigrant samples.

\section{Methods}

\section{Participants}

Chinese immigrant mothers $\left(N=202, M_{\text {age }}=37.56\right.$ years, $\left.S D=4.32\right)$ with preschool children $\left(M_{\text {age }}=4.56\right.$ years, $S D=1.17,45.5 \%$ females $)$ and Korean immigrant mothers $(N=$ $131, M_{\text {age }}=35.61$ years, $\left.S D=3.85\right)$ with preschool children $\left(M_{\text {age }}=4.24\right.$ years, $S D=1.03,55.7 \%$ females) participated in the present study. Almost all the mothers (98.5\%) were first-generation immigrants, and most had attained a college or higher degree (85.9\%). The average time of residence of the mothers in the United States was 11 years $(S D=6.98)$, ranging from 2 months to 35 years. No differences in mother's age, education, nationality, children's age, gender, and their length of residence in the U.S. were found between the two groups.

\section{Procedure}

Mothers were recruited from local language schools, churches, supermarkets and libraries in the Maryland and Washington, D.C. area. All assessments were conducted during a home visit in mothers’ preferred language (English, Mandarin, or Korean). After providing written consent 
for their participation, mothers completed the PAQ and a demographic measure that assessed the mother's age, education, nationality, time of residence in the U. S., and the child's age and gender. The families received $\$ 40$ and project newsletters as compensation for their participation. The University Institutional Review Board provided ethical approval for the study.

\section{Measures}

Mothers reported their attributions for daily parenting tasks on the PAQ. The PAQ was forward and back-translated by bilinguals who were fluent in Chinese and English or Korean and English to ensure that the back-translated questionnaires were comparable with the original English version. Most mothers chose to complete the measure in their heritage language (93\% of the Chinese immigrant mothers and $82 \%$ of the Korean immigrant mothers). Five causes (mothers' ability, mood and effort, task difficulty, and children's cooperation) contained in the PAQ are used to explain mothers' success and failure in seven parenting tasks (dressing, bathing, comforting, teaching, disciplining, communicating, and playing). An example of a successful event item is, "When I am able to get my child to take a bath, it is because: (a) I am good at this, (b) This is easy to do, (c) My child makes this easy to do, (d) I've tried hard, and (e) I'm in a good mood.” An example of an unsuccessful item for teaching is, "When I am unable to teach my child something new, it is because: (a) I am not good at this, (b) This is hard to do, (c) My child makes this hard to do, (d) I haven't tried hard enough, and (e) I'm in a bad mood.” For each item, parents rated each cause on a scale from 1 (not at all a reason) to 5 (very much a reason). Ten subscales are generated by adding up the scores for each cause across the seven tasks separately for parenting success and failure.

\section{Data Analysis Plan}


The aggregated rating for each cause across the seven parenting tasks was computed separately for success and failure, representing a tendency to attribute parenting success or failure to a particular reason as a continuous variable.. Three participants who completed very few items (less than 20\% of the 14 items) in the measure were excluded from the analysis. At least 5 of the 7 items of a particular cause were required in order to calculate a sum score for the cause; otherwise, the data was considered missing for that specific cause. This approach resulted in $0.7 \%$ of missing data in our sample. Little's MCAR test $\left(\chi^{2}=111.095, d f=106, p=.35\right)$ suggested that the data were missing completely at random. The full-information maximum likelihood method (Enders, 2013) was used to handle missing data.

To confirm the factor structure of the PAQ in the Asian immigrant sample, multi-group confirmatory factor analyses were conducted for attributions of success and failure separately using the lavaan package (Rosseel, 2012) in R. First, three competing models (see Figure 1), the unidimensional structure model, the internal-external structure model, and the controllable-uncontrollable structure model, were tested in the Chinese immigrant sample and the Korean immigrant sample simultaneously to test configural invariance. The model with the best fit was retained. Second, based on the final model from the previous step, metric and scalar invariance of the PAQ were tested. Partial invariance was tested when full invariance was not established. Maximum Likelihood estimator was used because the data were aggregated and considered continuous. Overall model fit was evaluated using various fit indices including the Satorra-Bentler robust scaled chi-square statistic $\left(\mathrm{S}-\mathrm{B} \chi^{2}\right)$, the comparative fit index (CFI), the root mean squared error of approximation (RMSEA), and the standardized root mean square residual (SRMR). CFI above .95, RMSEA below .06, and SRMR below .08 indicated a good model fit; whereas CFI above .90, RMSEA below .08, and SRMR below .10 were required for 
an acceptable model fit (Brown, 2014). McDonald's omega and $\Delta$ CFI were used to evaluate the reliability of the latent factors and the measurement invariance, respectively. $\Delta C F I$ smaller than -.01 was required to establish invariance between different groups (Cheung \& Rensvold, 2002).

\section{Results}

\section{Preliminary Analyses}

The descriptive statistics and correlations for all variables examined were presented in Table 1. For both the Chinese immigrant and the Korean immigrant samples, all correlations between causes for parenting successes were significant, and all correlations between causes for parenting failures were significant except for one (correlation between ability and child cooperation causes for parenting failure in the Chinese sample). Several correlations between causes across parenting successes and failures were also significant ( 9 of 25 in the Chinese sample and 6 of 25 in the Korean sample), and most of the coefficients of these correlations were within a small magnitude (Cohen, 1988). Moreover, previous research has demonstrated that maternal attributions for parenting success and failure should be investigated separately (Bugental et al., 1998; Bornstein et al., 2017). Therefore, analyses were conducted separately for the parenting success and failure attributions.

\section{CFA Results}

Configural invariance. The unidimensional structure, internal-external structure, and controllable-uncontrollable structure (see Figure 1) of the PAQ were analyzed for both success and failure items separately. For the models of success attributions, the configural model of the unidimensional structure indicated a poor model fit according to all indices $\left(\chi^{2}=\right.$ 190.27, $p<.00$, CFI $=.71$, RMSEA $=.33$, SRMR $=.13$ ); the configural model of the internal-external structure was also a poor fit $\left(\chi^{2}=178.22, p<.00\right.$, CFI $=.72$, RMSEA $=.36$, 
SRMR = .12). However, all indices for the configural model of the controllable-uncontrollable structure fit the data notably better than the other two models $\left(\chi^{2}=17.36, p=.03\right.$, CFI $=.99$, RMSEA $=.08$, SRMR $=.03$, see Table 2 ). As shown in Table 3, each item in the controllable-uncontrollable model significantly loaded on its factor, with factor loadings ranging from .56 to 1.05 in the Chinese immigrant group, .61 to 1.02 in the Korean immigrant group. The correlations between the controllable factor and uncontrollable factor were .45 $(p<.01)$ and .50 $(p<.01)$ for the Chinese immigrant group and the Korean immigrant group, respectively. For the Chinese immigrant group, the AVE (average variance extracted) was .78 for the uncontrollable factor and .56 for the controllable factor; for the Korean immigrant group, the AVE was .84 for the uncontrollable factor and .62 for the controllable factor.

Similar results were found for failure attributions. Poor model fit was indicated for the configural model of the unidimensional structure $\left(\chi^{2}=147.25, p<.00\right.$, CFI $=.78$, RMSEA $=.29, \mathrm{SRMR}=.11)$ and the configural model of the internal-external structure $\left(\chi^{2}=\right.$ 146.92, $p<.00$, CFI $=.78$, RMSEA $=.32$, SRMR $=.11)$. In contrast, the configural model of the controllable-uncontrollable structure $\left(\chi^{2}=13.83, p=.10\right.$, CFI $=.99$, RMSEA $=.07$, SRMR $=.03$, see Table 2) fits the data better than the other two models. Each item in the controllable-uncontrollable model significantly loaded on its respective factor, with factor loadings ranging from .50 to .93 in the Chinese immigrant group, .76 to .92 in the Korean immigrant group (see Table 3). The correlations between the controllable factor and uncontrollable factor were .36 $(p<.01)$ and $.39(p<.001)$ for the Chinese immigrant group and the Korean immigrant group, respectively. For the Chinese immigrant group, the AVE (average variance extracted) was .72 for the uncontrollable factor and .55 for the controllable factor; for 
the Korean immigrant group, the AVE was .75 for the uncontrollable factor and .70 for the controllable factor.

Metric and scalar invariance. Because the controllability-based structure yielded better model fit than the other two structures in the configural invariance test, metric and scalar invariances were examined based on the controllable-uncontrollable factor structure.

Model fits of metric invariance were acceptable. After constraining the factor loadings to the same, the Chi-square difference test was not statistically significant for success attribution $\left(\Delta \chi^{2}=0.88, \Delta d f=3, p=.83\right)$ and the $\Delta \mathrm{CFI}$ was -.01 , indicating that metric invariance was established (Cheung \& Rensvold, 2002). After imposing equality constraints on factor loadings for failure attribution, the chi-square difference test was significant $\left(\Delta \chi^{2}=10.45, \Delta d f=3, p\right.$ $=.02$ ) but the $\triangle \mathrm{CFI}$ was smaller than -.01, within the suggested range of invariance (Cheung $\&$ Rensvold, 2002). Therefore, we deemed metric invariance was established for both success and failure attributions. See the model fit indices in Table 2.

Model fits of scalar invariance were poor (see Table 2) and thus efforts were made to establish partial scalar invariance. After the equality constraints for intercepts of effort and child cooperation causes were relaxed in the success attribution model, the chi-square difference test was significant $\left(\Delta \chi^{2}=14.36, \Delta d f=3, p<.01\right)$, but the $\Delta$ CFI became smaller than -.01. After three of the five causes (effort, mood, and child cooperation) were freely estimated in the failure attribution model, the chi-square difference test was significant $\left(\Delta \chi^{2}=4.26, \Delta d f=2, p=.04\right)$, but the $\triangle \mathrm{CFI}$ also indicated an adequate level of invariance (smaller than -.01). Therefore, partial scalar invariance was established for both success and failure attributions. See the model fit indices in Table 2.

\section{Reliability}


To examine the reliability of the PAQ measure for the Asian immigrant sample, the Cronbach's alpha of the questionnaire and its 10 subscales were computed for the Chinese immigrant and Korean immigrant samples. The reliability score for the overall PAQ was .76 for the Chinese immigrant sample and .78 for the Korean immigrant sample. The 10 subscales also proved reliable for both the Chinese immigrant sample (reliability scores ranging from .82 - .90) and the Korean immigrant sample (reliability scores ranging from .87 - .93). As indicated by our results, the PAQ and its subscales were reliable for the Asian immigrant sample.

McDonald's omega of the four PAQ dimensions suggested by the CFA results were .71 (controllable success), .87 (uncontrollable success), .79 (controllable failure), and .83 (uncontrollable failure) in the Chinese immigrant sample, and .83(controllable success), .91 (controllable success), .87 (controllable failure), and .86 (uncontrollable failure) in the Korean immigrant sample.

\section{Discussion}

\section{Factor Structure}

The present study provided empirical evidence for a controllability-based factor structure of the PAQ, rather than a unidimensional structure or a locus-based structure (see Figure 1), in Chinese and Korean immigrant samples. One possible explanation for the superiority of the controllability-based factor structure is the focus on parental control among Asian parents (Cheung \& Pomerantz, 2011; Ng et al., 2014). According to Weiner’s attribution theory (Weiner et al., 1971), causes referring to one’s ability and the difficulty of the task are perceived as constant and stable. In contrast, causes referring to one’s effort and mood are perceived to be variable and changeable over time. Due to the relative constancy of causes attributed to personal ability and task difficulty, which pertain to innate qualities of the person and task, and the 
variability of causes attributed to personal mood and effort invested in a particular situation, the former causes are more likely to be considered uncontrollable and the latter controllable (Weiner, 1985). Consistent with this idea, Asian immigrant mothers' ability to bathe their child, for example, and the difficulty of this task fell on one factor whereas the causes of mothers' effort and mood fell on another factor in the present study. Therefore, the two factors were labelled as uncontrollable parental attributions and controllable parental attributions, respectively. These findings supported our hypothesis that Asian immigrant parents would be more likely to make attributions regarding their parenting successes and failures based on the controllability of the causes rather than locus of the causes, which may reflect their Confucian-based cultural valuing of parental control (Pomerantz \& Wang 2009).

Consistent with the idea that parents’ attributions regarding their parenting successes and failures should be explored separately (Bornstein \& Cote, 2004; Bornstein, Putnick, \& Lansford, 2011), the correlations of parents' attributions to a specific cause across success and failure were relatively low except for the attributions to child cooperation (.54) and parents' mood (.66). Studies have found that Asian mothers made fewer attributions to their ability or task difficulty during parenting success, and more attributions to their efforts during parenting failure because of their general emphasis on fulfilling parenting responsibilities through their ability and efforts (Bornstein \& Cote, 2004; Bornstein et al., 2011). However, child cooperation and parents’ mood may not be as central to their evaluation of their own performances during a certain parenting task. Therefore, attributions to these two causes may not vary as much as attributions to parents’ effort and ability across parenting successes and failures.

Another interesting finding of the present study was that attributions about the child's cooperation as a cause of parenting success and failure loaded on the controllable factor rather 
than the uncontrollable factor. In previous research within Western contexts, child characteristics or behaviors as causal explanations in the PAQ was derived from the "luck" attribution (Weiner et al., 1971), in which child cooperation is described as external, unstable, and uncontrollable (Sirignano \& Lachman, 1985). Our finding that attributions of child cooperation loaded on the same factor as mothers' effort and mood, which are considered controllable, may be due to traditional Asian cultural childrearing beliefs that parents can have a major impact on children's behaviors (Chao, 2001; Ng et al., 2014).

Asian parents highly value the importance of an integrated, cohesive, and interdependent family (Triandis, 2001). Parents’ responsibility for shaping children’s behavior and children’s compliance are two fundamental components that uphold the family structure. Thus, parents’ major tasks include providing proper parenting and creating optimal environments for their children, which are more likely achieved through strong control over the situation during parent-child interactions. Moreover, filial piety, one of the most important traditional Confucian values, emphasizes children’s respect and obedience to their parents as well as intimate connection with their parents (Ho, 1994; Kim, 2006). Thus, for Asian families, children’s level of cooperativeness may be more likely to be perceived as controllable.

Previous research found that Chinese and Korean mothers make more external attributions regarding their children's behaviors compared to European immigrant mothers based on the locus-based structure (e.g., Cheah \& Rubin, 2004; Cheah \& Park, 2006). Although these previous findings may seem to contradict our current findings, they are both in fact consistent with the Asian cultural emphasis on parental control and creation of an ideal childrearing environment. More external attributions are made for children's behaviors because Chinese and Korean mothers attach greater importance to environmental influences on their children's 
behaviors, particularly parenting (Cheah \& Rubin, 2003; Park \& Cheah, 2006). Similarly, the controllability dimension is emphasized in attributions regarding parent-child interactions since parental control is crucial during caregiving in achieve optimal outcomes. Thus, our finding of the significance of the controllability dimension in maternal attributions for parent-child interactions echoes previous findings of greater endorsement of external influences in maternal attributions for children’s behaviors among Chinese and Korean mothers.

\section{Measurement Invariance}

The present study further focused on the attributional patterns of Chinese and Korean immigrant mothers, and established measurement invariance between two Asian immigrant groups. Specifically, metric invariance was successfully established between Chinese and Korean immigrant mothers for success and failure attributions, which revealed mothers' similar focus on the controllability of each individual cause. Overall, mothers from China and Korea share some similar childrearing values and parenting beliefs due to their Confucian historical roots (Chao, 2000; Sung, 2010), which may account for their similar general controllability-based attribution patterns regarding successful and unsuccessful parenting practices.

In addition, partial scalar invariance was successfully established in the present study, which revealed a similar baseline level across the groups regarding the tendency to attribute parenting success and failure to uncontrollable causes (ability and task difficulty). However, the present study also found that Chinese immigrant mothers displayed a higher baseline of attributing parenting success and failure to controllable causes (mood, effort, and child cooperation) than Korean immigrant mothers. Despite the shared cultural background of Confucian ideologies, Korean mothers are proposed to be more influenced by Western ideologies regarding childrearing values and parenting cognitions due to their longer access to 
Western ideas and beliefs regarding parenting issues (Ju \& Chung, 2000; Park \& Cheah, 2005). Chinese immigrant mothers may view their children and the parent-child interactions as more malleable and exert more effort to change the parenting outcome (Ng et al., 2014), which account for their higher baseline level of attributing successful and unsuccessful caregiving outcomes to controllable causes. Korean immigrant mothers, on the other hand, may be more likely to view their children as independent individuals due to the Western influence (Cote, Kwak, Putnick, Chung, \& Bornstein, 2015; Park \& Cheah, 2005), and display relatively lower baseline level of attributing caregiving outcomes to controllable causes.

\section{Reliability}

Our results revealed that the PAQ and its subscales were reliable for Chinese immigrant mothers and Korean immigrant mothers. Moreover, the reliability estimates for the controllability-based factors in the present study $(\alpha=.82-.93)$ were generally more stable than those found in previous studies ( $\alpha=$.63-.95; Bornstein \& Cote, 2003; Sirignano \& Lachman, 1985), which may be due to the great emphasis on children's need and daily parental care as indirect ways of expressing warmth among Asian immigrant parents (Cheah, Li, Zhou, Yamamoto, \& Leung, 2015). Therefore, the PAQ and its controllability-based factors have potential utility in future research on Asian immigrant mothers.

\section{Limitations and Further Directions}

Several important limitations of the current study should be noted. First, almost all the mothers in our study were first-generation, middle-class immigrants. Therefore, our findings may not generalize to Asian immigrant mothers of second or later generations. Future research should seek to better understand Asian immigrant parents’ cognitions across different socioeconomic statuses and generations. Moreover, the hypotheses in this study were formulated based on 
previous research on attributions of Chinese and Korean mothers in their countries of origin. However, further study is needed to explore and confirm the controllability-based factor structure of the PAQ within Chinese and Korean samples in their countries of origin to further understand the role of culture in parental attributions.

The focus of this study was on Asian immigrants. We had proposed that our findings reflected the stronger focus on parental control in Asian compared to Western cultures reported in previous research. However, the controllability dimension has been shown to be an important dimension in Western parents’ attribution as well (Bugental et al., 1998). Thus, future studies should compare the competing factor structures of controllability and locus of the PAQ in Western parents.

\section{Conclusions}

The present study found that the controllability-based structure fit the data better than the unidimensional or locus-based structure of parental attributions. Metric and partial scalar invariance of the controllability-based model were established between Chinese and Korean immigrant mothers. Our findings revealed controllability as a key dimension in East Asian immigrant mothers’ parent-referent attributions. Thus, studies investigating Asian parents’ cognitions should be conducted with careful consideration of the cultural context. The present study also indicated that practitioners working with Asian immigrant parents and children need to ensure that their practices and programs are culturally-informed. For example, programs could increase Asian parents' self-efficacy and confidence about their parenting through enhancing their feelings of controllability during daily caregiving practice, when appropriate. In addition to improving parenting skills, appropriate attention to culturally-relevant parental attributions may promote positive outcomes in Asian immigrant parents. 


\section{References}

Bartlett, M. S. (1950). Tests of significance in factor analysis. British Journal of Statistical Psychology, 3(2), 77-85. https://doi.org/10.1111/j.2044-8317.1950.tb00285.x

Bornstein, M. H., \& Cote, L. R. (2003). Cultural and parenting cognitions in acculturating cultures: 2. Patterns of prediction and structural coherence. Journal of Cross-Cultural Psychology, 34(3), 350-373. https://doi.org/10.1177/0022022103034003007

Bornstein, M. H., \& Cote, L. R. (2004). Mothers' parenting cognitions in cultures of origin, acculturating cultures, and cultures of destination. Child Development, 75(1), 221-235. https://doi.org/10.1111/j.1467-8624.2004.00665.x

Bornstein, M. H., Haynes, O. M., Azuma, H., Galperín, C., Maital, S., Ogino, M., ... \& Toda, S. (1998). A cross-national study of self-evaluations and attributions in parenting: Argentina, Belgium, France, Israel, Italy, Japan, and the United States. Developmental Psychology, 34(4), 662-676. https://doi.org/10.1037/0012-1649.34.4.662

Bornstein, M. H., \& Putnick, D. L. (2007). Chronological age, cognitions, and practices in European American mothers: A multivariate study of parenting. Developmental Psychology, 43(4), 850-864. https://doi.org/10.1037/0012-1649.43.4.850

Bornstein, M. H., Putnick, D. L., \& Lansford, J. E. (2011). Parenting attributions and attitudes in cross-cultural perspective. Parenting, 11(2-3), 214-237. https://doi.org/10.1080/15295192.2011.585568

Bornstein, M. H., Putnick, D. L., \& Suwalsky, J. T. (2017). Parenting cognitions $\rightarrow$ parenting practices $\rightarrow$ child adjustment? The standard model. Development and Psychopathology, 1-18. https://doi.org/10.1017/S0954579417000931

Brown, T. A. (2014). Confirmatory factor analysis for applied research. Guilford Publications. 
Bugental, D. B., Johnston, C., New, M., \& Silvester, J. (1998). Measuring parental attributions: Conceptual and methodological issues. Journal of Family Psychology, 12(4), 459-480. https://doi.org/10.1037/0893-3200.12.4.459

Chao, R. K. (1995). Chinese and European American cultural models of the self-reflected in mothers' childrearing beliefs. Ethos, 23(3), 328-354.

https://doi.org/10.1525/eth.1995.23.3.02a00030

Chao, R. K. (2000). The parenting of immigrant Chinese and European American mothers: Relations between parenting styles, socialization goals, and parental practices. Journal of Applied Developmental Psychology, 21(2), 233-248. https://doi.org/10.1016/S0193-3973(99)00037-4

Chao, R. K. (2001). Extending research on the consequences of parenting style for Chinese Americans and European Americans. Child Development, 72(6), 1832-1843. https://doi.org/10.1111/1467-8624.00381

Cheah, C. S., Li, J., Zhou, N., Yamamoto, Y., \& Leung, C. Y. (2015). Understanding Chinese immigrant and European American mothers’ expressions of warmth. Developmental Psychology, 51(12), 1802. https://doi.org/10.1037/a0039855

Cheah, C. S., \& Park, S. Y. (2006). South Korean mothers’ beliefs regarding aggression and social withdrawal in preschoolers. Early Childhood Research Quarterly, 21(1), 61-75. https://doi.org/10.1016/j.ecresq.2006.01.004

Cheah, C. S., \& Rubin, K. H. (2003). European American and mainland Chinese mothers' socialization beliefs regarding preschoolers' social skills. Parenting: Science and Practice, 3(1), 1-21. https://doi.org/10.1207/S15327922PAR0301_01 
Cheah, C., \& Rubin, K. (2004). European American and Mainland Chinese mothers' responses to aggression and social withdrawal in preschoolers. International Journal of Behavioral Development, 28(1), 83-94. https://doi.org/10.1080/01650250344000299

Cheung, C. S. S., \& Pomerantz, E. M. (2011). Parents’ involvement in children’s learning in the United States and China: Implications for children’s academic and emotional adjustment. Child Development, 82(3), 932-950.

https://doi.org/10.1111/j.1467-8624.2011.01582.x

Cheung, G. W., \& Rensvold, R. B. (2002). Evaluating goodness-of-fit indexes for testing measurement invariance. Structural Equation Modeling, 9(2), 233-255. https://doi.org/10.1207/S15328007SEM0902_5

Cohen, J. (1992). Statistical power analysis. Current Directions in Psychological Science, 1(3), 98-101. https://doi.org/10.1111/1467-8721.ep10768783

Cote, L. R., Kwak, K., Putnick, D. L., Chung, H. J., \& Bornstein, M. H. (2015). The acculturation of parenting cognitions: A comparison of South Korean, Korean immigrant, and European American mothers. Journal of Cross-Cultural Psychology, 46(9), 1115-1130. https://doi.org/10.1177/0022022115600259

Dix, T. H., \& Grusec, J. E. (1985). Parent attribution processes in the socialization of children. Parental belief systems: The psychological consequences for children, 201-233.

Enders, C. K. (2013). Dealing with missing data in developmental research. Child Development Perspectives, 27, 27-31. https://doi.org/10.1111/cdep.12008

Enlund, E., Aunola, K., \& Nurmi, J. E. (2015). Stability in parents' causal attributions for their children's academic performance: A nine-year follow-up. Merrill-Palmer Quarterly, 61(4), 509-536. https://doi.org/10.13110/merrpalmquar1982.61.4.0509 
Ho, D. Y. F. (1986). Chinese patterns of socialization: A critical review. In M. H. Bond (Ed.), The psychology of the Chinese people (pp. 1-37). New York: Oxford University Press.

Ho, D. Y. F. (1994). Filial piety, authoritarian moralism, and cognitive conservatism in Chinese societies. Genetic, Social, and General Psychology Monographs, 120(3), 347-365.

Hu, L. T., \& Bentler, P. M. (1999). Cutoff criteria for fit indexes in covariance structure analysis: Conventional criteria versus new alternatives. Structural equation modeling: $a$ multidisciplinary journal, 6(1), 1-55. https://doi.org/10.1080/10705519909540118

Humes, K., Jones, N. A., \& Ramirez, R. R. (2011). Overview of race and Hispanic origin, 2010. US Department of Commerce, Economics and Statistics Administration, US Census Bureau.

Ju, D.-B., \& Chung, I.-H. (2000). The effect of maternal employment on schoolchildren’s educational aspirations in Korea. Journal of Research in Childhood Education, 15, 1-20. https://doi.org/10.1080/02568540009594773

Kaiser, H. F. (1974). An index of factorial simplicity. Psychometrika, 39(1), 31-36. https://doi.org/10.1007/BF02291575

Kim, K.-W. (2006). Hyo and Parenting in Korea. In K. H. Rubin \& O. B. Chung (Eds.), Parenting beliefs, behaviors, and parent-child relations: A cross-cultural perspective (pp. 207-222). New York: Psychology Press.

Lee, E. G., \& Han, S. Y. (2017). The Effects of Mothers' Parental Internal Attributions and Neuroticism on Self-Perception of the Parental Role. Korean Journal of Child Studies, 38(1), 49-62. https://doi.org/10.5723/kjcs.2017.38.1.49

MacPhee, D., Seybold, J., \& Fritz, J. Parental attributions and self-esteem among mothers of delayed children. Unpublished manuscript, University of Colorado. 
Miller, S. A. (1995). Parents' attributions for their children's behavior. Child Development, 66(6), 1557-1584. https://doi.org/10.1111/j.1467-8624.1995.tb00952.x

Ng, F. F. Y., Pomerantz, E. M., \& Deng, C. (2014). Why are Chinese mothers more controlling than American mothers? “My child is my report card”. Child Development, 85(1), 355-369. https://doi.org/10.1111/cdev.12102

Park, S. Y., \& Cheah, C. S. (2005). Korean mothers’ proactive socialisation beliefs regarding preschoolers’ social skills. International Journal of Behavioral Development, 29(1), 24-34. https://doi.org/10.1080/01650250444000306

Park, Y. S., Kim, B. S., Chiang, J., \& Ju, C. M. (2010). Acculturation, enculturation, parental adherence to Asian cultural values, parenting styles, and family conflict among Asian American college students. Asian American Journal of Psychology, 1(1), 67-79. https://doi.org/10.1037/a0018961

Pomerantz, E. M., \& Wang, Q. (2009). The role of parental control in children's development in Western and East Asian countries. Current Directions in Psychological Science, 18(5), 285-289. https://doi.org/10.1111/j.1467-8721.2009.01653.x

Rosseel, Y. (2012). lavaan: An R Package for Structural Equation Modeling. Journal of Statistical Software, 48(2), 1 - 36. https://doi.org/10.18637/jss.v048.i02

Sawrikar, V., \& Dadds, M. (2018). What Role for Parental Attributions in Parenting Interventions for Child Conduct Problems? Advances from Research into Practice. Clinical Child and Family Psychology Review, 21(1), 41-56. https://doi.org/10.1007/s10567-017-0243-4

Sigel, I. E., McGillicuddy-DeLisi, A. V., \& Goodnow, J. J. (Eds.). (2014). Parental belief systems: The psychological consequences for children. Psychology Press. https://doi.org/10.2307/353190 
Sirignano, S. W., \& Lachman, M. E. (1985). Personality change during the transition to parenthood: The role of perceived infant temperament. Developmental Psychology, 21(3), 558-567. https://doi.org/10.1037/0012-1649.21.3.558

Snarr, J. D., Slep, A. M. S., \& Grande, V. P. (2009). Validation of a new self-report measure of parental attributions. Psychological Assessment, 21(3), 390-401. https://doi.org/10.1037/a0016331

Sung, H. Y. (2010). The influence of culture on parenting practices of East Asian families and emotional intelligence of older adolescents: A qualitative study. School Psychology International, 31(2), 199-214. https://doi.org/10.1177/0143034309352268

Triandis, H. C. (2001). Individualism and collectivism: Past, present, and future. The Handbook of Culture and Psychology, (pp. 35-50). New York, NY, US: Oxford University Press.

Vandenberg, R. J., \& Lance, C. E. (2000). A review and synthesis of the measurement invariance literature: Suggestions, practices, and recommendations for organizational research. Organizational Research Methods, 3(1), 4-70. https://doi.org/10.1177/109442810031002

Weiner, B. (1985). An attributional theory of achievement motivation and emotion. Psychological Review, 92(4), 548-573. https://doi.org/10.1037/0033-295X.92.4.548

Weiner, B., Frieze, I. H., Kukla, A., Reed, L., Rest, S., \& Rosenbaum, R. M. (1971). Perceiving the causes of success and failure. Morristown, NJ: General Learning Press.

Woolfson, L. M., Taylor, R. J., \& Mooney, L. (2011). Parental attributions of controllability as a moderator of the relationship between developmental disability and behaviour problems. Child: Care, Health and Development, 37(2), 184-194. https://doi.org/10.1111/j.1365-2214.2010.01103.x 
Yirmiya, N., \& Weiner, B. (1986). Perceptions of controllability and anticipated anger. Cognitive Development, 1(3), 273-280. https://doi.org/10.1016/S0885-2014(86)80005-3 
Table 1

Descriptive Statistics in the Chinese Immigrant and the Korean Immigrant Samples

\begin{tabular}{|c|c|c|c|c|c|c|c|c|c|c|c|c|}
\hline & 1 & 2 & 3 & 4 & 5 & 6 & 7 & 8 & 9 & 10 & $M$ & $S D$ \\
\hline 1. Ability success & - & $.81 * *$ & $.50 * *$ & $.41 * *$ & $.41 * *$ & -.05 & .02 & $-.18 *$ & -.15 & -.10 & 19.27 & 6.60 \\
\hline 2. Difficulty success & $.70 * *$ & - & $.36 * *$ & $.38 * *$ & $.34 * *$ & -.06 & -.07 & .14 & $-.17 *$ & -.11 & 17.87 & 6.41 \\
\hline 3. Effort success & $.33 * *$ & $.16^{*}$ & - & $.51 * *$ & $.74 * *$ & .09 & .11 & .14 & .07 & $.19 *$ & 22.10 & 5.63 \\
\hline 4. Child success & $.34 * *$ & $.18 * *$ & $.38 * *$ & - & $.51 * *$ & -.05 & -.01 & .02 & $.24^{*}$ & .11 & 23.77 & 5.63 \\
\hline 5. Mood success & $.31 * *$ & $.27 * *$ & $.53 * *$ & $.38 * *$ & - & .09 & .01 & $.18^{*}$ & .17 & $.41 * *$ & 21.48 & 6.86 \\
\hline 6. Ability failure & .08 & $.15^{*}$ & .11 & .07 & .12 & - & $.69 * *$ & $.56 * *$ & $.49 * *$ & $.59 * *$ & 14.27 & 5.27 \\
\hline 7. Difficulty failure & $.18 *$ & $.21 * *$ & .12 & .09 & .10 & $.71 * *$ & - & $.49 * *$ & $.54 * *$ & $.47 * *$ & 16.76 & 5.68 \\
\hline 8. Effort failure & -.03 & -.13 & .18 & $.23 *$ & $.29 * *$ & $.23 * *$ & $.24 * *$ & - & $.63 * *$ & $.77 * *$ & 17.78 & 6.14 \\
\hline 9. Child failure & -.01 & -.05 & $.16^{*}$ & $.54 * *$ & .12 & .13 & $.25 * *$ & $.28 * *$ & - & $.67 * *$ & 18.65 & 6.84 \\
\hline 10. Mood failure & .01 & -.06 & .17 & $.22 * *$ & $.66^{* *}$ & $.17 * *$ & $.23 * *$ & $.55^{* *}$ & $.29 * *$ & - & 17.51 & 7.45 \\
\hline$M$ & 21.26 & 18.61 & 25 & 25.82 & 20.76 & 13.03 & 13.87 & 20.93 & 24.5 & 20.01 & & \\
\hline$S D$ & 6.60 & 6.60 & 5.44 & 5.23 & 6.79 & 5.51 & 5.27 & 6.03 & 5.80 & 6.91 & & \\
\hline
\end{tabular}


Note: The $M$ s and SDs for the Chinese immigrant sample are presented below the diagonal and for the Korean immigrant sample are presented below the diagonal.

Table 2

Fit Indices for Two-Group CFA Models of Success and Failure Attributions

\begin{tabular}{|c|c|c|c|c|c|c|c|}
\hline Model & Structure & S-B $\chi^{2}$ & $d f$ & CFI & $\Delta \mathrm{CFI}$ & RMSEA & SRMR \\
\hline \multicolumn{8}{|l|}{ Success } \\
\hline Configural & Unidimensional & 190.27 & 10 & .71 & - & .33 & .13 \\
\hline Configural & Internal-external & 178.22 & 8 & .72 & - & .36 & .12 \\
\hline Configural & Controllable-uncontrollable & 17.36 & 8 & .99 & - & .08 & .03 \\
\hline Metric & Controllable-uncontrollable & 18.24 & 11 & .98 & -.01 & .06 & .03 \\
\hline Scalar & Controllable-uncontrollable & 71.55 & 16 & .91 & -.08 & .15 & .09 \\
\hline Partial scalar ${ }^{\mathrm{a}}$ & Controllable-uncontrollable & 32.60 & 14 & .97 & -.01 & .08 & .05 \\
\hline
\end{tabular}

Failure

$\begin{array}{llllllll}\text { Configural } & \text { Unidimensional } & 147.25 & 10 & .78 & .29 & .11 \\ \text { Configural } & \text { Internal-external } & 146.92 & 8 & .78 & - & .32 & .11\end{array}$


ASIAN IMMIGRANT PARENTAL ATTRIBUTION

Configural Controllable-uncontrollable

Metric Controllable-uncontrollable

Scalar Controllable-uncontrollable

Partial scalar $^{\mathrm{a}} \quad$ Controllable-uncontrollable
13.83

24.28

70.74

28.54
8

11

16

13
29

.98

$-.01$

.08

.22

.08

.00

.07

a. The intercepts of effort and child cooperation causes were freely estimated for success attribution; the intercepts of effort, mood, and child cooperation causes were freely estimated for failure attribution. 
Table 3

Results of the Configural Invariance Model of the Controllable-Uncontrollable Structure

\begin{tabular}{|c|c|c|c|c|c|c|}
\hline & \multicolumn{3}{|c|}{ Chinese Immigrant } & \multicolumn{3}{|c|}{ Korean Immigrant } \\
\hline & Intercept & $\begin{array}{l}\text { Unstandardized } \\
\text { Factor Loading }\end{array}$ & $\begin{array}{l}\text { Standardized } \\
\text { Factor Loading }\end{array}$ & Intercept & $\begin{array}{l}\text { Unstandardized } \\
\text { Factor Loading }\end{array}$ & $\begin{array}{l}\text { Standardized } \\
\text { Factor Loading }\end{array}$ \\
\hline \multicolumn{7}{|c|}{ Success Attribution: Uncontrollable } \\
\hline Ability & 21.26 & 1.00 & 1.05 & 19.27 & 1.00 & 1.02 \\
\hline Task Difficulty & 18.62 & .64 & .67 & 17.87 & .75 & .79 \\
\hline \multicolumn{7}{|c|}{ Success Attribution: Controllable } \\
\hline Effort & 24.99 & 1.00 & .73 & 22.10 & 1.00 & .90 \\
\hline Child Cooperation & 25.81 & .74 & .56 & 23.78 & .68 & .61 \\
\hline Mood & 20.80 & 1.21 & .70 & 21.48 & 1.11 & .82 \\
\hline \multicolumn{7}{|c|}{ Failure Attribution: Uncontrollable } \\
\hline Ability & 13.00 & 1.00 & .76 & 14.21 & 1.00 & .92 \\
\hline Task Difficulty & 13.84 & 1.17 & .93 & 14.80 & 1.01 & .82 \\
\hline \multicolumn{7}{|c|}{ Failure Attribution: Controllable } \\
\hline Effort & 20.90 & 1.00 & .74 & 17.70 & 1.00 & .86 \\
\hline Child Cooperation & 24.47 & .71 & .50 & 18.61 & .99 & .76 \\
\hline Mood & 20.08 & 1.17 & .76 & 17.35 & 1.25 & .89 \\
\hline
\end{tabular}

Note. All parameters were statistically significant at .001 level. 


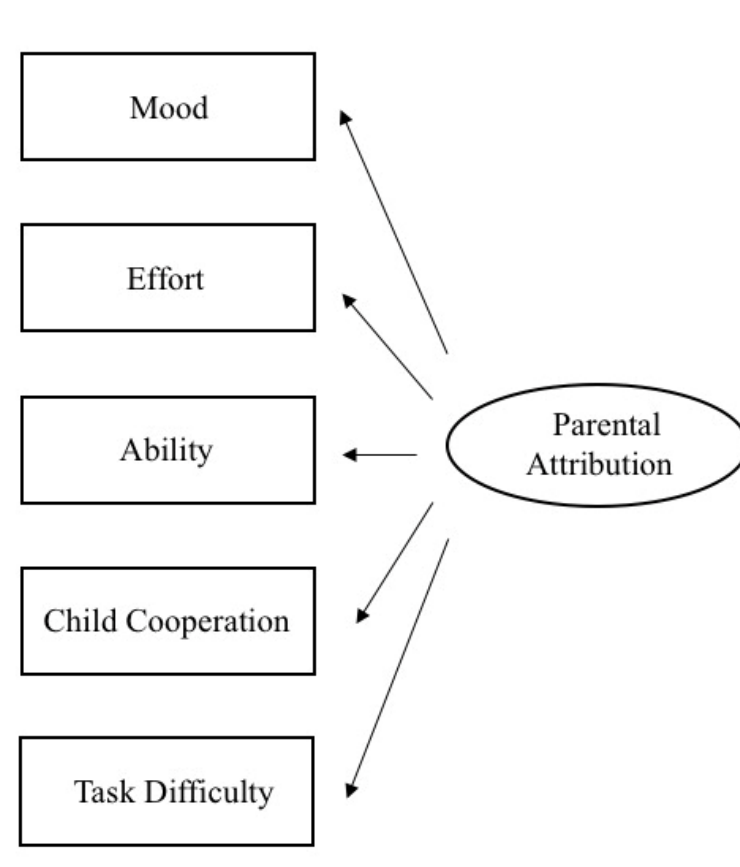

Unidimensional Model

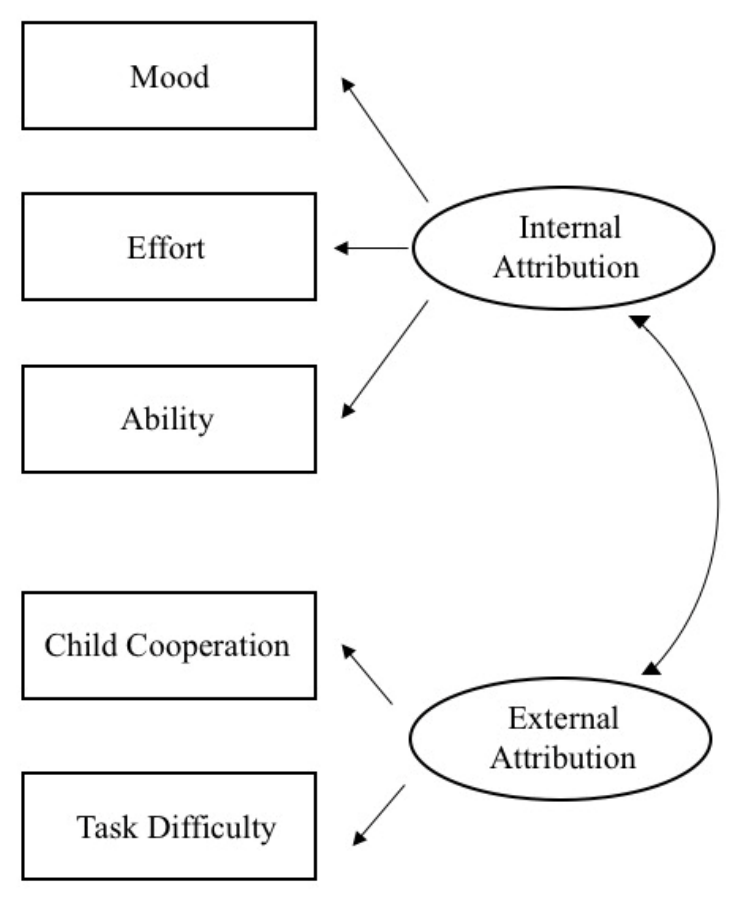

Internal-External Model

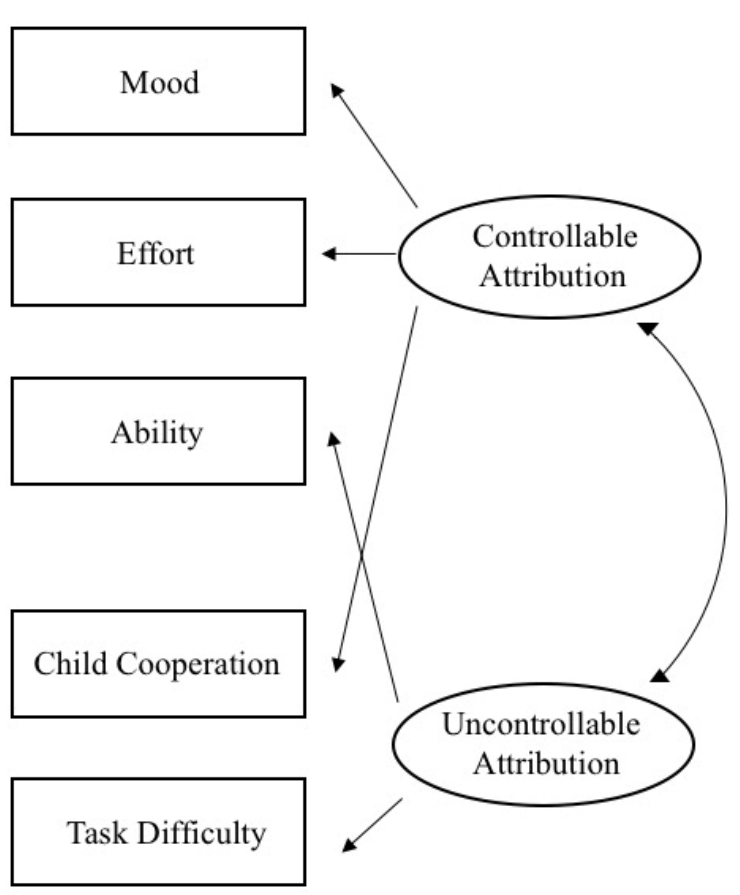

Controllable-Uncontrollable Model

Figure 1. Factor structures of the controllable-uncontrollable, internal-external, and unidimensional models. Measurement errors were omitted for simplicity. 